\title{
On the non-linearities of ship's restoring and the Froude-Krylov wave load part
}

\author{
Jerzy Edward Matusiak
}

Aalto-University, School of Science and Technology, Applied Mechanics Department, Espoo, Finland

\begin{abstract}
When formulating a general, non-linear mathematical model of ship dynamics in waves the hydrostatic forces and moments along with the Froude-Krylov part of wave load are usually concerned. Normally radiation and the diffraction forces are regarded as linear ones. The paper discusses briefly few approaches, which can be used in this respect. The concerned models attempt to model the non-linearities of the surface waves; both regular and the irregular ones, and the nonlinearities of the restoring forces and moments. The approach selected in the Laidyn method, which is meant for the evaluation of large amplitude motions in the 6 degrees-of-freedom, is presented in a bigger detail. The workability of the method is illustrated with the simulation of ship motions in irregular stern quartering waves.
\end{abstract}

KEY WORDS: Froude-Krylov pressures; Nonlinear restoring; Stern quartering seas.

\section{INTRODUCTION}

The method called Laidyn (Matusiak, 2000b, 2001) is meant for the evaluation of ship motions in waves. Ship is regarded as a rigid intact body. The mathematical model behind the method comprises the elements of maneuvering and makes allowance for the non-linear large amplitudes motions in waves. The original version, meant for the regular waves only, was based on the so-called two-stage approach. At the first stage of this approach, linear approximation to the rigid body motion in waves is evaluated. A number of nonlinearities involved in ship dynamics in waves are taken into account and the total response of ship in the six degrees-offreedom is solved at the second stage. In particular the nonlinearities of the rigid body dynamics, non-linear terms of the restoring and the Froude-Krylov forces, ship resistance, the forces developed by a propulsor and by a rudder are taken into account. Details of the method are given for instance in Matusiak (2007).

Extension of the method, aimed at dealing with the longcrested irregular waves, led to giving up the concept of the two-stage evaluation of the responses. Instead, a direct solution of ship response is evaluated in the time-domain. All other features of the method are preserved. Similarly as in the original method, the linear models represent the radiation forces and moments and also the diffraction part of the wave excitation acting on ship. It is worth noting that these forces and moments are oriented with the axes of the body-fixed coordinate system.

Corresponding author: Jerzy Edward Matusiak e-mail: Jerzy.Matusiak@tkk.fi
A linear surface wave theory of Airy is used to model surface waves. However, in order to take non-linearities of the Froude-Krylov loads into account, both the wetted surface of ship's hull and pressures are evaluated up to the actual position of free surface. This is done using a kinematical model involving a simple summation of the undisturbed component waves and knowing the position of a hull in space. Extrapolation of pressures beyond the linear model of Airy can in principle be done in two different ways. These are presented and discussed further in the paper

\section{FORMULATION}

For the sake of this paper completeness, a short description of the Laidyn method is presented in the following. A more detailed description can be found in the abovementioned

\section{Equations of motion}

Equations of ship rigid motions are given by a set of six expressions (1) given below (Matusiak, 2007) with $u, v$ and $w$ being the projections of the velocities of ship's centre of gravity in the Earth-fixed inertial co-ordinate system on the axes of the moving body-fixed system. The angular position of the ship is given by so-called modified Euler's angles denoted as $\psi, \theta$ and $\phi$. Refer to Fig. 1 for the definitions of the inertia and body-fixed co-ordinate systems. In equations 1 , $X_{\mathrm{g}}, Y_{\mathrm{g}}, Z_{\mathrm{g}}, K_{\mathrm{g}}, M_{\mathrm{g}}$ and $N_{\mathrm{g}}$ depict the components of global reaction force and moment vectors acting on the ship. These are given in the-body fixed co-ordinate system $x y z . m$ and $I_{\mathrm{ij}}$ 
mean ship's mass and the components of the mass moment of inertia.

$$
\begin{aligned}
& X_{g}-m g \sin \theta=m(\dot{u}+Q w-R v) \\
& Y_{g}+m g \cos \theta \sin \phi=m(\dot{u}+R v-P w) \\
& Z_{g}-m g \cos \theta \cos \phi=m(\dot{u}+P v-Q u) \\
& K_{g}=I_{x} P-I_{x y} Q-I_{x y} R+\left(I_{x} R-I_{z x} P-I_{z y} Q\right) Q \\
& \quad-\left(I_{y} Q-I_{y z} R-I_{y x} P\right) R \\
& M_{g}=-I_{y x} \dot{P}-I_{y} \dot{Q}-I_{y z} \dot{R}+\left(I_{x} P-I_{x y} Q-I_{x y} R\right) R \\
& \quad-\left(I_{z} R-I_{z x} P-I_{z y} Q\right) P \\
& N_{g}=-I_{z x} \dot{P}-I_{z y} \dot{Q}-I_{z} \dot{R}+\left(I_{y} Q-I_{y z} R-I_{y x} P\right) P \\
& \quad-\left(I_{x} P-I_{x y} Q-I_{x z} R\right) Q
\end{aligned}
$$

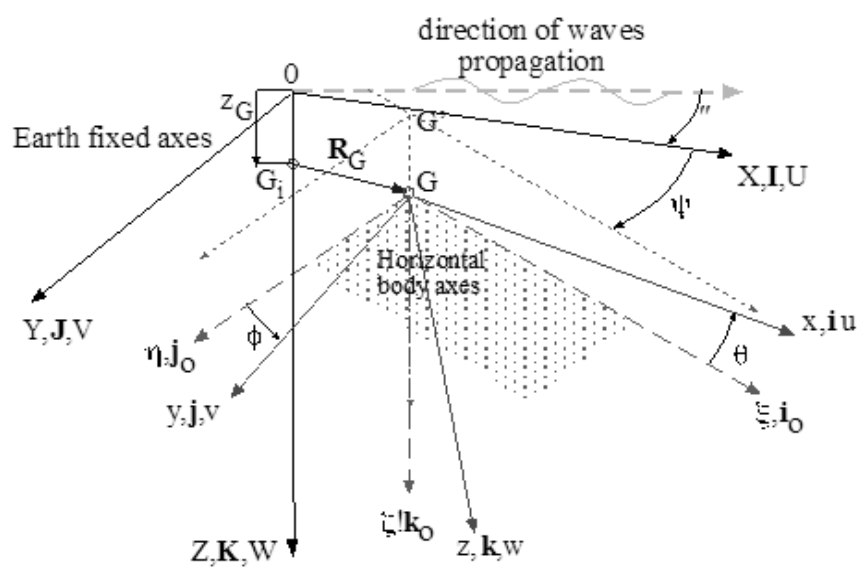

Fig. 1 Co-ordinate systems used to describe ship motion (Matusiak, 2007)

The relation between the velocities of the ship's centre of gravity in the inertial co-ordinate system and their projections $u, v$ and $w$ on the axes of the moving body-fixed system is (Fossen, 1994; Clayton and Bishop, 1982)

$$
\left\{\begin{array}{l}
\dot{X}_{G} \\
\dot{Y}_{G} \\
\dot{Z}_{G}
\end{array}\right\}=\left[\begin{array}{ccc}
\cos \psi \cos \theta & \cos \psi \cos \phi & \cos \psi \sin \theta \cos \phi \\
& -\sin \psi \cos \phi & +\sin \psi \sin \phi \\
\sin \psi \cos \theta & \sin \psi \sin \theta \sin \phi & \sin \psi \sin \theta \cos \phi \\
-\sin \theta & -\cos \psi \cos \phi & -\cos \psi \sin \phi \\
& -\cos \theta \sin \phi & \cos \theta \cos \phi
\end{array}\right]\left\{\begin{array}{l}
u \\
v \\
w
\end{array}\right\}
$$

Moreover a relation between the angular velocity vector $\Omega=P \mathbf{i}+Q \mathbf{j}+R \mathbf{k}$ and the Euler's angles $\psi, \theta$ and $\phi$ is needed (Fossen, 1994; Clayton and Bishop, 1982)

$$
\left\{\begin{array}{l}
\dot{\phi} \\
\dot{\theta} \\
\dot{\psi}
\end{array}\right\}=\left[\begin{array}{ccc}
1 & \sin \phi \tan \theta & \cos \phi \tan \theta \\
0 & \cos \phi & -\sin \phi \\
0 & \sin \phi / \cos \theta & \cos \phi / \cos \theta
\end{array}\right]\left\{\begin{array}{l}
P \\
Q \\
R
\end{array}\right\}
$$

\section{Numerical Solution}

Equations of motion 1 are solved numerically using the 4th order Runge-Kutta integration scheme yielding velocities $u, v, w, P, Q$ and $R$ in the co-ordinate system fixed with the moving ship. Equations 2 and 3 are used to integrate theses velocities into the ship's position in the inertial (Earth-fixed) co-ordinate system.

At each time step the components of global reaction force and moment vectors acting on the ship $X_{\mathrm{g}}, Y_{\mathrm{g}}, Z_{\mathrm{g}}, K_{\mathrm{g}}, M_{\mathrm{g}}$ and $N_{\mathrm{g}}$ have to be given. These include restoring, radiation and wave forces, ship resistance, the forces developed by a propulsor and a rudder. These are described in bigger detail in Matusiak (2001, 2002). An allowance for wind loading is included as well.

\section{NON-LINEAR MODELS OF FROUDE-KRYLOV AND RESTORING FORCES AND MOMENTS}

\section{General on the non-linear models}

There are a number of different approaches used in taking into account nonlinearities associated with restoring and Froude-Krylov forces and moments in waves. Some of these are direct extensions to the static buoyancy models. In this approach a simple or more sophisticated model of static lever (GZ-curve) in waves is considered (Hong et al., 2009; VidicPerunovic, 2009; Bulian and Francescutto 2008). A parametric variation of restoring term results in a Mathieutype equation for roll motion, which in some cases gives a prediction of the so-called parametric roll resonance.

A multivariable Taylor expansion up to the third order can be used to model describe strongly coupled restoring terms of heave, roll and pitch. Also this approach is successfully used in a prediction of parametric roll resonance (Rodríguez et al., 2007).

Boundary element method, that is a panel method, either of a Rankine-type or utilizing a special Green function approach for unsteady free surface flows can be used in a linear or a non-linear form as well.

RANSE methods, that is the tools solving both the unsteady free surface flow problem using ReynoldsAveraged-Navier-Stokes equations and the body dynamics problem are already in use. As they are nearly free of any assumptions they may be regarded as the most sophisticated and reliable methods. However, their usage at present is mainly of a demonstrative nature only, as they require a lot of computer resources and take a lot of time to execute.

A more profound and detailed description of the methods used in predicting large amplitude motions in waves can be found in the reports of the ITTC Committees on Seakeeping and Stability.

In the next paragraph I will concentrate on a very restricted problem of modeling the non-linearities of hydrostatic and Froude-Krylov pressures in the panel-type seakeeping method. 
Non-linear hydrostatic and Froude-Krylov forces and moments in the context of a panel representation of ship hull

When considering ship motions in waves, it is commonly believed that the most important contributors to the nonlinearities in the external forces acting on a ship hull are the restoring and Froude-Krylov forces and moments. Evaluation of these is done using a wetted surface of ship hull represented by a discrete panel model. This takes into account both an instantaneous position of ship in space and pressure due to waves extending up to the actual water surface. This is done as follows.

Position of each control point $\mathrm{c}$, that is a centre point of a panel, of Fig. 2 below is transformed from the body-fixed coordinate system $\left(x_{\mathrm{c}}, y_{\mathrm{c}}, z_{\mathrm{c}}\right)$ to the inertial Earth-fixed system $\left(X_{\mathrm{c}}, Y_{\mathrm{c}}, Z_{\mathrm{c}}\right)$ using transformation given by the following formula which is similar to the transformation 2 .

$$
\begin{aligned}
\left\{\begin{array}{l}
X_{c} \\
Y_{c} \\
Z_{c}
\end{array}\right\}= & \left\{\begin{array}{l}
X_{G} \\
Y_{G} \\
Z_{G}
\end{array}\right\} \\
& {\left[\begin{array}{ccc}
\cos \psi \cos \theta & \cos \psi \cos \phi & \cos \psi \sin \theta \cos \phi \\
-\sin \psi \cos \phi & +\sin \psi \sin \phi \\
\sin \psi \cos \theta & \sin \psi \sin \theta \sin \phi & \sin \psi \sin \theta \cos \phi \\
-\sin \theta & -\cos \psi \cos \phi & -\cos \psi \sin \phi \\
& -\cos \theta \sin \phi & \cos \theta \cos \phi
\end{array}\right]\left\{\begin{array}{l}
x_{c} \\
y_{c} \\
z_{c}
\end{array}\right\} }
\end{aligned}
$$

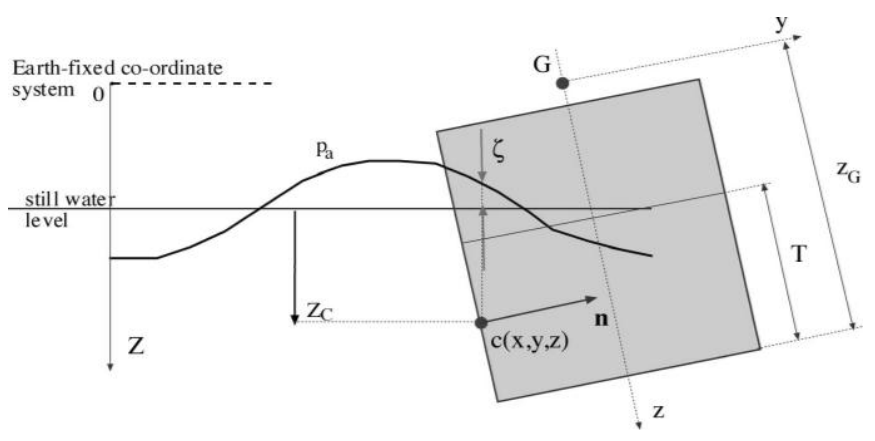

Fig. 2 Evaluation of hydrostatic and Froude-Krylov pressure.

Wave elevation above the control point $\mathrm{c}$ is given by a sum over the wave components $N$

$$
\zeta(t)=\sum_{i=1}^{N} A_{i} \cos \left[k_{i}\left(X_{c} \cos \mu-Y_{c} \sin \mu\right)+\omega_{i} t+\delta_{i}\right]
$$

where $A_{\mathrm{i}}$ and $k_{\mathrm{i}}=\omega_{1}^{2} / \mathrm{g}$ are wave amplitude and wave number corresponding to the $i$-th wave component. Phase angle $\delta_{\mathrm{i}}$ of each wave component is a random number. Details on generating wave trains from a given wave spectra are given for instance in Naito (1995) or Matusiak (2000a).

There are three models for evaluating the pressure at point $\mathrm{c}$. The first one is a linear Froude-Krylov pressure model with the wetted surface extending up to the still water level. It is worth noting that restoring forces and moments are taking non-linearity into account. In this model pressure is given by the expression

$$
p_{c}(t)=\rho g\left(\begin{array}{l}
Z_{c}+\sum_{i=1}^{N} A_{i} \exp \left(-k_{i} Z_{c}\right) \\
\times \cos \left[k_{i}\left(X_{c} \cos \mu-Y_{c} \sin \mu\right)-\omega_{i} t+\delta_{i}\right]
\end{array}\right)
$$

Two other models take into account the actual wetted surface. The pressures are evaluated for the immersed panels, that is for $Z_{\mathrm{c}}+\zeta(t)>0$.

The first of these models, which is presented in Faltinsen (1990), is similar to the one given by Formula 6 but with a linear extrapolation of pressure between the still water and actual water levels. Thus it can be understood as an extension of the linear model. The third model, called stretched pressure model, is given by the formula with the free surface raised by the amount of wave elevation (5) in the argument of the exponent function.

$$
p_{c}(t)=\rho g\left(\begin{array}{l}
Z_{c}+\sum_{i=1}^{N} A_{i} \exp \left(-k_{i} Z_{c}+\zeta(t)\right) \\
\times \cos \left[k_{i}\left(X_{c} \cos \mu-Y_{c} \sin \mu\right)-\omega_{i} t+\delta_{i}\right]
\end{array}\right)
$$

The forces $\mathbf{F}$ and moments $\mathbf{M}$ are obtained by integrating the pressure (6 or 7) in the body fixed co-ordinate system. This integration is performed numerically by summing up the contribution from each wetted panel using

$$
\begin{aligned}
\mathrm{F}_{\mathrm{F}, \mathrm{K}}^{\mathrm{total}} & =\sum_{i}^{M} \mathrm{~F}_{\mathrm{F}, \mathrm{K}, \mathrm{i}}^{\mathrm{total}}=\sum_{i}^{M} \rho_{i} \Delta S_{i} n_{i} \\
\mathrm{~F}_{\mathrm{F}, \mathrm{K}}^{\mathrm{total}} & =\sum_{i}^{M} \mathrm{r}_{i} \times \mathrm{F}_{\mathrm{F}, \mathrm{K}, \mathrm{i}}^{\mathrm{total}}
\end{aligned}
$$

where the total number of the panels is denoted by $M, \Delta S_{\mathrm{i}}$ is panel area, $\boldsymbol{n}_{\mathrm{i}}$ unit vector normal to panel and $\boldsymbol{r}_{\mathrm{i}}$ the position vector of the control point in the body-fixed co-ordinate system $x y z$.

\section{SHIP BEHAVIOR IN IRREGULAR WAVES USING THREE DIFFERENT MODELS OF FROUDE- KRYLOV PRESSURE}

Ship motions in irregular waves were evaluated using the above-described three models of Froude-Krylov pressures. The investigated vessel is the one used in the benchmark study initiated by the International Towing Tank Conference and presented in (Spanos and Papanikolaou, 2009). This is a containership of waterline length of $L_{\mathrm{PP}}=150 \mathrm{~m}$. Metacentric height was set to $G M_{0}=1.2[\mathrm{~m}]$ yielding the natural roll period of $T_{\phi}=21[s]$. A model of this ship was also investigated earlier in a similar study (ITTC, 2002). 
In all three cases the same operational condition was set. The desired significant wave height was set to $H_{\mathrm{S}}=5[\mathrm{~m}]$ and period $T_{1}=7.3[s]$. In order to save the computing time, wave spectrum was represented by $N=19$ wave components only. Ship was set to the stern quartering long-crested waves at heading $\mu=30[\mathrm{deg}]$. In simulations ship is propelled with a propeller and steered with a rudder under a $P D$-control. Ship operated for 40 minutes in each numerical test. Summary of the results is presented in Table 1 below.

Each of the irregular realization of waves was different due to a randomness built into the waves' generation algorithm. Heave, roll and pitch motion components, as computed in time-domain, are presented in terms of their standard deviations, maxima and minima.

Table 1 Summary of the results

\begin{tabular}{|c|c|c|c|c|c|c|c|}
\hline FK-linear & Wave $[m]$ & Heave $[m]$ & Heave (linear) & Roll [deg] & Roll (linear) & Pitch $[m]$ & Pitch (linear) \\
\hline stdev & 1.15 & 0.20 & 0.19 & 3.99 & 1.09 & 0.58 & 0.64 \\
\hline $\operatorname{Max}$ & 2.98 & 0.46 & 0.46 & 11.65 & 3.40 & 1.44 & 1.36 \\
\hline Min & -3.62 & -0.52 & -0.46 & -9.78 & -3.10 & -0.98 & -1.43 \\
\hline Faltinsen & Wave $[m]$ & Heave $[m]$ & Heave (linear) & Roll [deg] & Roll (linear) & Pitch $[m]$ & Pitch (linear) \\
\hline stdev & 1.33 & 0.27 & 0.26 & 4.04 & 1.16 & 0.76 & 0.87 \\
\hline $\operatorname{Max}$ & 3.21 & 0.56 & 0.55 & 12.34 & 3.43 & 1.64 & 1.71 \\
\hline Min & -3.19 & -0.69 & -0.58 & -11.20 & -3.63 & -1.47 & -1.71 \\
\hline Stretched & Wave $[m]$ & Heave $[m]$ & Heave (linear) & Roll [deg] & Roll (linear) & Pitch $[m]$ & Pitch (linear) \\
\hline stdev & 1.14 & 0.19 & 0.18 & 2.63 & 1.09 & 0.49 & 0.56 \\
\hline $\operatorname{Max}$ & 3.16 & 0.46 & 0.44 & 6.94 & 3.47 & 1.35 & 1.43 \\
\hline Min & -3.60 & -0.45 & -0.43 & -6.82 & -2.94 & -1.10 & -1.37 \\
\hline
\end{tabular}

Linear approximation to the global responses of ship in irregular waves is evaluated in order to judge the effects of non-linearity on the derived responses. Normally, in the linear seakeeping theory, a constant forward speed is assumed. In the Laidyn method surge motion of ship is evaluated in the time domain taking into account amongst the others propeller action and variations of the wetted surface. Thus in-plane motion of ship is simulated in time-domain along with the other motion components. This results in ship position $X_{G}, Y_{\mathrm{G}}$ in the Earth-fixed co-ordinate system. This and the knowledge of transfer functions of the corresponding responses make it possible to evaluate linear approximation of the responses using the expressions

$$
\begin{aligned}
& z_{L}(t)=\sum_{i=1}^{N} A_{i} z_{L 0} \cos \left[k_{i}\left(X_{G} \cos \mu-Y_{G} \sin \mu\right)-\omega_{i} t+\delta_{i}-\gamma_{z i}\right] \\
& \phi_{L}(t)=\sum_{i=1}^{N} k_{i} A_{i} \phi_{L 0} \cos \left[k_{i}\left(X_{G} \cos \mu-Y_{G} \sin \mu\right)-\omega_{i} t+\delta_{i}-\gamma_{\phi i}\right] \\
& \theta_{L}(t)=\sum_{i=1}^{N} k_{i} A_{i} \theta_{L 0} \cos \left[k_{i}\left(X_{G} \cos \mu-Y_{G} \sin \mu\right)-\omega_{i} t+\delta_{i}-\gamma_{\theta i}\right]
\end{aligned}
$$

where terms with subscripts $L O$ depict gain factors of the transfer functions and $\gamma$ corresponding phase angles The transfer functions were obtained with the software based on linear seakeeping theory (Journee, 1992).

There are no significant differences between the results obtained with different models for Froude-Krylov pressures, except for the roll motion. Time domain simulations with Laidyn give much higher roll angles than the linear frequency domain strip theory. The selected operational conditions that is a combination of wave period, heading and ship speed yield frequently a resonant roll motion, which is visible in the selected time histories of the responses presented in Figs. 3, 4 and 5. Simulated case is critical for the ship in this particular case because of a kind of focusing effect of waves.

The encounter period of majority of waves is very close to the natural period of roll. As a result a resonant roll motion develops frequently during the simulation period.

The selected records represent maxima of roll motion in irregular waves for each model of Froude-Krylov pressure. Built-up of a roll develops for the wave groups having the encounter period close to the natural period of roll. The same cannot be seen with a fully linear frequency domain analysis. Linear solution relates roll angle to an instantaneous value of wave slope and thus it does not have relation to wave grouping. It is worth noting that roll damping was kept same valued in all models.

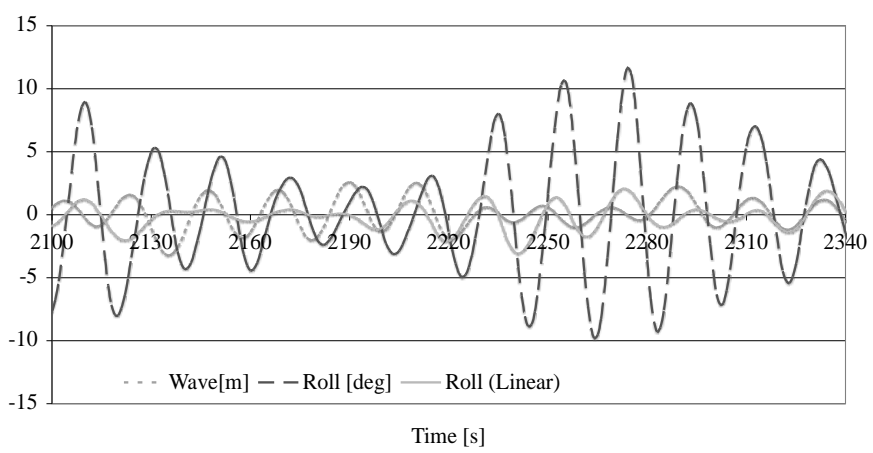

Fig. 3 Built-up of a resonant roll motion in irregular stern quartering waves simulated using a linear Froude-Krylov pressure model. 


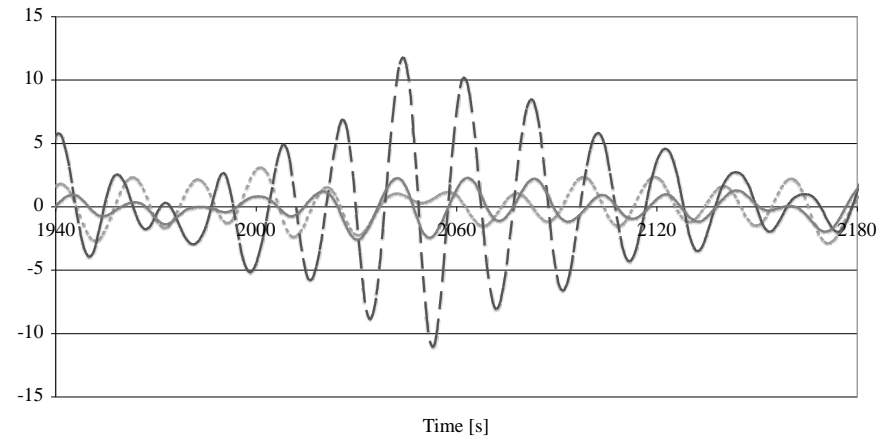

Fig. 4 Built-up of a resonant roll motion in irregular stern quartering waves simulated using the wave pressure model of Faltinsen.

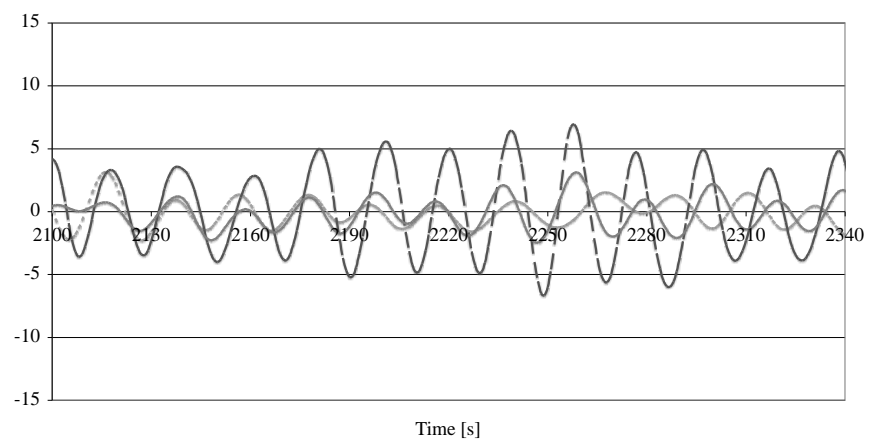

Fig. 5 Built-up of a resonant roll motion in irregular stern quartering waves simulated using stretched wave pressure model.

\section{CONCLUSIONS}

It is impossible to draw a conclusion which of the models used in the presented time-domain simulations is best one. For the investigated case, all three yield much higher roll angles than the ones evaluated by the fully linear frequency domain model. A development of roll resonance for the investigated situation is known from the literature (Kluwe and Krüger, 2007) and acknowledged by the Authorities (IMO, 1995, 2006). A further research is needed to validate the method. In particular model tests in both regular and also in irregular quartering waves will provide better validation data for the method.

\section{REFERENCES}

Bulian, G. and Francescutto, A., 2008. Theoretical prediction and experimental verification of multiple steady states for parametric roll. In Proceedings of the 10th International Workshop on Ship Stability and Operational Safety, Daejeon, Korea.

Clayton, B.R. and Bishop, R.E.D., 1982. Mechanics of marine vehicles. London, 1982, ISBN 0419 12110-2.

Faltinsen, O. M., 1990 Sea loads on ships and offshore structures. Cambridge University Press, New York, 328 p.

Fossen, T.I., 1994. Guidance and control of ocean vehicles. John. Wiley and Sons Ltd., ISBN 0471941131.
IMO, 1995. Guidance to the master for avoiding dangerous situations in following and quartering seas. MSC/ Circ.707/19.10.

IMO, 2006. Draft revised guidance to the master for avoidance dangerous situations in adverse weather and sea conditions. SLF 49/WP. 2 ANNEX 2.

ITTC, 2002. 23rd International Towing Tank Conference, Report of the Specialist Committee of Extreme Ship Motions and Capsizing. Venice, Italy, 8-14 September 2002.

Journee, J.M. 1992. Strip theory algorithms. Report MEMT 24, Delft University of Technology, Ship Hydrodynamics Laboratory, Nederland.

Kluwe, F. and Krüger, S., 2007. Using full-scale capsizing accidents for the validation of numerical; Seakeeping Simulations. In Proceedings of the 9th International Ship Stability Workshop, Hamburg, Germany.

Matusiak, J., 2000a. Dynamics of cargo shift onboard a ship in irregular beam waves. International Shipbuilding Progress, 47(449), pp. 77-93.

Matusiak, J., 2000b. Two-stage approach to determination of non-linear motions of ship in waves. $4^{\text {th }}$ Osaka Collouqium on Seakeeping Performance of Ships, Osaka, Japan, 17-21 October 2000.

Matusiak, J., 2001. Importance of memory effect for capsizing prediction. $5^{\text {th }}$ International workshop University of Trieste, Universitá di Trieste, Italy, 12-13 September 2001.

Matusiak, J., 2002. Two-stage approach to determination of large amplitude motions of a rigid ship in waves. 15th Nordic Seminar on Computational Mechanics, Aalborg, Denmark, 18-19 October 2002.

Matusiak, J., 2007. On certain types of ship response disclosed by the two-stage approach to ship dynamics. Archives of Civil and Mechanical Engineering, 7(4), pp. 151-166.

Naito, S., 1995. Generation and absorption of waves. symposium on wave generation, analysis and related problems in experimental tanks- especially on directional wave. Wave generation's 95, Yokohama National University, Japan, 25 September 1995. pp. 1-27.

Rodríguez, C.A. Holden, C. Perez, T. Drummen, I. Neves, M. A.S. and Fossen, T.I., 2007. Validation of a container ship model for parametric rolling. In Proceedings of the 9th International Ship Stability Workshop, Hamburg, Germany. 30-31 August 2007.

Hong, S.Y. Yu, H.C. Kim, S. and Sung, H.G., Investigation of parametric roll of a container ship in irregular seas by numerical simulation, Proceedings of the 10th International Conference on Stability of Ships and Ocean Vehicles, St. Petersburg, Russia, 21-26 June 2009. pp. 549-558.

Spanos, D. and Papanikolaou, A., 2009. Benchmark study on numerical simulation methods for the prediction of parametric roll of ships in waves, Proceedings of 10th International Conference on Stability of Ships and Ocean Vehicles, St. Petersburg, Russia, 21-26 June 2009. pp. 549558.

Vidic-Perunovic, J., 2009. Effect of roll restoring lever calculation on parametric roll prediction in a stationary sea state, Proceedings of 10th International Conference on Stability of Ships and Ocean Vehicles, St. Petersburg, Russia, 21-26 June 2009, pp. 549-558. 